\title{
The role of the separation point in streamwise vortex-induced vibrations
}

\author{
Neil Cagneya,b, Stavroula Balabani ${ }^{\mathrm{b}, *}$ \\ ${ }^{a}$ School of Engineering and Materials Science, Queen Mary University of London, \\ London E1 $4 N S$, UK \\ ${ }^{b}$ Department of Mechanical Engineering, University College London, WC1E 6BT, UK
}

\begin{abstract}
Structures in crossflow are susceptible to vortex-induced vibrations (VIV) when the vortex-shedding becomes synchronised with the structural vibration. Strategies to control VIV often include modifying the separation point on the cylinder surface, such as adding helical strakes, although there remains disagreement regarding the mechanism by which these work. We explore the role of the separation point on VIV acting in the streamwise (drag) direction, by performing high-speed Particle-Image Velocimetry (PIV) measurements of the wake and the structural displacement of a range of cylinders with different cross-sectional shapes, including circular (no fixed separation points), equilateral triangles (fixed separation points) and elliptical cylinders (which act as an intermediate case). None of the non-circular cylinders are found to exhibit VIV, despite having approximately the same experimental conditions (mass ratio, structural damping, Reynolds number range, etc.) as the circular cylinders, which undergo VIV. The phase-averaged PIV measurements of the near wake of the circular cylinder are used to calculate the separation angle throughout the shedding cycle for different wake modes, and it is shown that all the wake modes that are associated with VIV require a periodic movement of the separation point. In contrast, the variation in the separation angle was negligible for the von Kármán vortex street observed behind near-stationary circular cylinders and for all non-circular cylinders. The experiments illustrate the great sensitivity of the wake mode and streamwise VIV to modifications of the separation point and demonstrate that even a moderately elliptical cylinder (major to minor axis ratio of 1.54) is sufficient
\end{abstract}

*s.balabani@ucl.ac.uk 
to completely suppress VIV.

Keywords:

\section{Introduction}

Vortex-induced vibrations (VIV) occur when the natural frequency of a structure in crossflow coincides with the frequency of fluid forcing due to vortex-shedding, leading to a complex coupling between the structural motion and wake dynamics. VIV of a cylinder is a classical fluid-structure interaction problem, affecting a wide range of areas of industry and nature, including heat exchanges, offshore structures, chimneys (Blevins, 1977) and vegetation (Levy and Liu, 2013). In recent years, there has also been growing interest in the use of VIV as a source of renewable energy (Bernitsas et al., 2008).

Vortex-induced vibrations are controlled by the reduced velocity, $U_{r}=$ $U_{0} / f_{n} D$ (where $U_{0}$ is the freestream velocity, $f_{n}$ is the natural frequency of the structure in still fluid and $D$ is the diameter of the structure) and the Strouhal number, $S t=f_{v s} D / U_{0}$ (where $f_{v s}$ is the vortex-shedding frequency for a stationary cylinder). Vibrations tend to occur when the forcing coincides with the natural frequency. As the fluctuations in the drag force occur at twice the shedding frequency, VIV in the streamwise (drag) direction occurs at $U_{r} S t \sim 0.5$.

The majority of VIV research in the literature has focussed on cylinders free to move only in the transverse (lift) direction, as the vibrations in this direction tend to be an order of magnitude greater than those in the streamwise direction (Griffin and Ramberg, 1976). However, there has been a renewed interest in streamwise VIV since the failure of the Monju reactor, in which design engineers failed to consider the risk of streamwise VIV, which occurs at a lower freestream velocity (Nakamura et al., 2001; Okajima et al., 2004). There has also been an interest in how the freedom to move in the streamwise direction affects the overall response of structures with multiple degrees-of-freedom (DOF) (Jauvtis and Williamson, 2003, 2004) and the resulting fatigue damage (Odahara et al., 2005).

The response regime of a cylinder free to move only in the streamwise direction has been studied by Aguirre (1977) (summarised by Naudascher (1987)), Nakamura et al. (2001), Okajima et al. (2004) and Cagney and Balabani $(2013 \mathrm{c}, \mathrm{a})$, and has been found to be similar to that observed for 


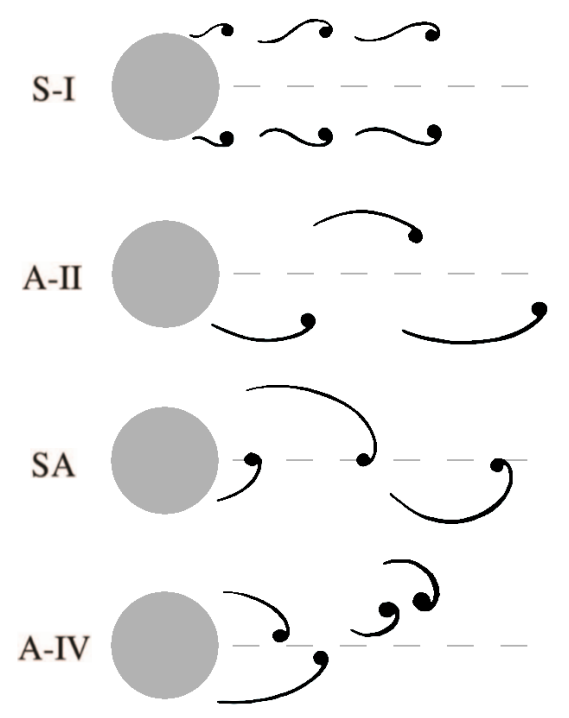

Figure 1: Sketch of the various wake modes observed behind a cylinder free to move in the streamwise direction.

cylinders with freedom to move in both the streamwise and transverse direction (Jauvtis and Williamson, 2003, 2004; Cagney and Balabani, 2014). The regime is characterised by two response branches, each with a peak vibration amplitude of $A / D \sim 0.1$. The first branch occurs over the range $U_{r} S t \approx 0.25-0.5$, and is associated with the symmetric shedding of vortices (Naudascher, 1987; Okajima et al., 2004; Konstantinidis and Balabani, 2007). This condition is known as the S-I wake mode, following the naming convention of Ongoren and Rockwell (1988), and is sketched in Figure 1. The symmetric arrangement of vortices is unstable, with vortices rearranging into an alternate structure downstream, which in turns leads to mode competition (Konstantinidis et al., 2007; Cagney and Balabani, 2013a), and the shedding reverts to an alternate mode at the peak of the first branch, i.e. a slightly modified form of the A-II mode which is observed behind a stationary cylinder (Figure 1), but with a shorter recirculation and vortex-formation lengths (Cagney and Balabani, 2013c).

The second response branch occurs over $U_{r} S t \approx 0.5-0.7$ and can occur in multiple forms depending on whether two or four vortices are shed per cycle (Cagney and Balabani, 2013b), which are referred to as the SA and 
A-IV modes, respectively (Figure 1). The SA mode is a special case of the A-II mode, in which the vortices are stronger, form very close to the cylinder and remain along the wake centreline as they convect downstream (Jauvtis and Williamson, 2003; Cagney and Balabani, 2013c).

The two branches are separated by a low amplitude region at $U_{r} S t \approx 0.5$. This paradoxical reduction in amplitude at the point where the fluid forcing coincides with the natural frequency has been variously hypothesised to arise from the competition between wake modes (Naudascher, 1987), changes in the phase of the fluid forcing and vortex-shedding (Nishihara et al., 2005; Konstantinidis et al., 2005) and a reduction in the amplitude of the unsteady forcing due to three-dimensionality in the wake (Cagney and Balabani, 2016).

The location of the separation point is intricately linked with the vortexshedding process and known to play an important role in VIV (Zdravkovich, 1981; Sui et al., 2016). This connection is utilised in VIV-suppression strategies such as the installation of helical strakes, which act as fixed separation points at various points along the span (Zdravkovich, 1981; Sui et al., 2016), although the exact mechanism by which this approach works is not fully understood (Bearman and Brankovicć, 2004).

Some early studies of VIV examined the streamwise response of cylinders with non-circular cross sections (see review of Naudascher (1987)), showing a tendency for bodies with sharp corners (aligned such that they are symmetrical about the wake centreline) to exhibit a reduced VIV response. The majority of studies of VIV of cylinders with fixed separation points have focussed on the response of structures that are free to move in the transverse direction, or multi-DOF cylinders over the reduced velocity range that is dominated by transverse VIV $\left(U_{r} S t \gtrsim 1\right)$.

Sen and Mittal (2011) used two-dimensional numerical simulations at low Reynolds numbers to study the response of a square cylinder and found that the lock-in range and peak transverse amplitude was reduced compared to a circular cylinder under the same conditions. Leontini and Thompson (2013) used a similar approach to examine the wake of a square cylinder aligned at $45^{\circ}$ to the freestream (i.e. in a diamond configuration) that was free to move in both the streamwise and transverse directions. They controlled sharpness of the separation points at either side of the cylinder by varying the curvature of the cylinder corners, and showed that when the corner was sharp (and the separation point was effectively fixed) the structural displacement signals became more irregular and there was an increase in the lock-in range and a slight increase in the vibration amplitude. Similar trends were observed 
in the experiments of Bokaian and Geoola (1984) for a square cylinders free to move only in the transverse direction with different corner radii at higher Reynolds numbers $(R e=400-7000)$.

Ajith Kumar et al. (2009) investigated the role of the separation point in VIV by forcing square cylinders with different corner curvatures to oscillate in the transverse direction, and found that for the cases with sharp corners, the vortex-shedding became disorganised and lock-in was disrupted. The tendency for fixed separation points to promote lock-in for diamond shaped cylinders but reduce the lock-in range and periodicity of VIV for square cylinders may be related to the after-body shape and the potential for shear layer reattachment (Chen and Liu, 1999; Nemes et al., 2012).

The sharpness of the separation point can also be controlled by using elliptical cylinders, in which the sharpness of the separation point can be increased by increasing the ratio of the transverse and streamwise cylinder diameters, such that the cylinder becomes less streamlined. Navrose et al. (2014) and Hasheminejad and Jarrahi (2015) examined the response of 2DOF cylinders at low $R e$ over the transverse response region $\left(U_{r} S t \gtrsim 1\right)$ and found that the vibration amplitude of a bluff ellipse was greater than that of a circular cylinder. A recent study by Leontini et al. (2018) found that for moderately elliptical cylinder (with a ratio of transverse to streamwise diameter of 1.5) with low mass ratio, the lock-in range was significantly extended relative to the case of an elliptical cylinder aligned with the major axis in the flow direction.

It is clear that the sharpness of the separation points has a strong effect on the fluid excitation experienced by structures in cross flow, although this effect does not appear to be consistent for all geometries (square, diamond, ellipse etc.); it is not clear what role the separation point plays at low reduced velocities, where VIV occurs primarily in the streamwise direction. In order to address these issues, we examine a range of cylinders that are symmetrical about the wake centreline and are free to move in the streamwise direction, including circular cylinders (no fixed separation points), triangular cylinders (clear fixed separation points at the sharp corners) and elliptical cylinders with the major axis aligned perpendicular to the flow (such that the fixity of the separation point can be varied by varying the ratio of the major and the minor axes). We chose to examine triangular rather than square cylinders, so as to reduce the potential effects of shear layer reattachment, which may obscure the role of the initial separation point. The details of the test facilities, cylinder properties and experimental procedure are outlined in the 


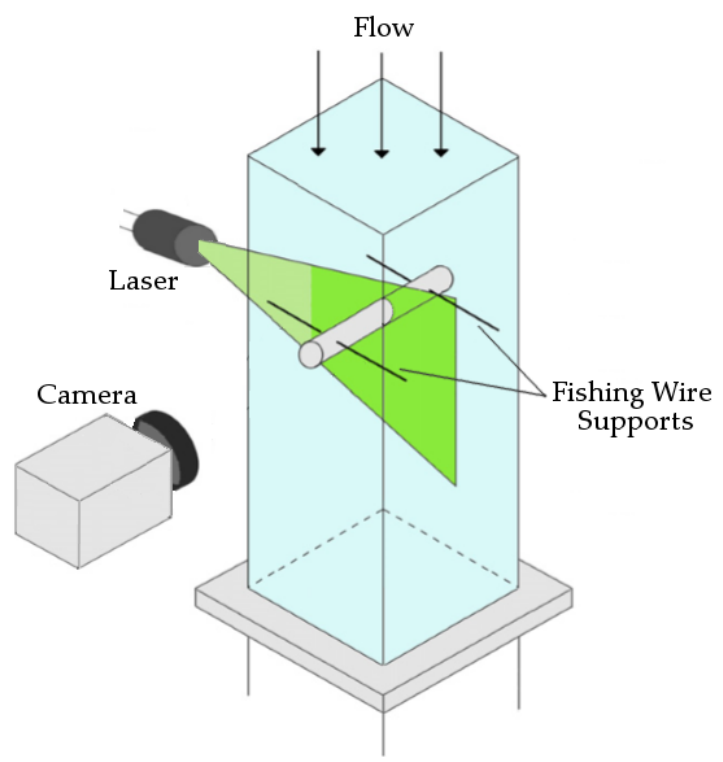

Figure 2: Schematic of the test-section used, including PIV measurement system and fishing wire supports.

following section, the results for the triangular and elliptical cylinders are presented and their significance for streamwise VIV are discussed in Sections 3.1 and 3.2, respectively, and finally some concluding remarks are presented in Section 4.

\section{Experimental Details}

The experiments were performed in a closed loop water tunnel, containing an acrylic test-section with a $72 \times 72 \mathrm{~mm}$ cross-sectional area, as has been described in Cagney and Balabani (2013c). A cooling system was used to hold the water temperature constant at $22 \pm 0.5^{\circ} \mathrm{C}$.

The various cylinders were suspended within the flow using fishing wire, such that they were free to move only in the streamwise direction, as shown in Figure 2. The stiffness of the structure was found to be linear and tests were performed to ensure that any rotational (pitching) motion was negligible. Tap tests were performed in still water and air to measure the natural frequency and structural damping ratio, $\zeta$, respectively. The physical properties of the various cylinders and some measurement settings are listed in 
Table 1: Summary of the experimental details of the PIV measurements of the different geometry cylinders. See text for a definition of the different symbols.

\begin{tabular}{lcccccccc} 
Geometry & $m^{*}$ & $f_{n}[\mathrm{~Hz}]$ & $\zeta$ & $D[\mathrm{~mm}]$ & $\epsilon$ & $L / D$ & $R e$ range & pixels $/ \mathrm{mm}$ \\
\hline $\begin{array}{l}\text { Circle } \\
\quad \text { Triangle }\end{array}$ & 1.17 & 23.7 & 0.0037 & 7.1 & 1 & 10 & $780-5620$ & 30.54 \\
$\begin{array}{c}\text { (Upstream- } \\
\text { pointing) } \\
\quad \text { Triangle }\end{array}$ & 1.4 & 15.54 & 0.0029 & 7.2 & $\mathrm{n} / \mathrm{a}$ & 9.86 & $750-5540$ & 24.87 \\
$\begin{array}{c}\text { (Downstream- } \\
\quad \text { pointing) }\end{array}$ & 1.4 & 22.66 & 0.0045 & 7.2 & $\mathrm{n} / \mathrm{a}$ & 9.86 & $370-4130$ & 24.65 \\
Circle & 0.98 & 15.24 & 0.0054 & 6.8 & 1 & 10.44 & $520-3440$ & 21.92 \\
Ellipse & 0.98 & 16.79 & 0.0055 & 6.858 & 1.54 & 10.35 & $550-4350$ & 21.35 \\
Ellipse & 0.98 & 20.1 & 0.0039 & 6.854 & 1.87 & 10.36 & $600-4520$ & 21.23
\end{tabular}

Table 2. In each case the structural damping ratio is low, in the range of $0.0029-0.0055$. The maximum Reynolds number $\left(R e=U_{0} D / \nu\right.$, where $\nu$ is the kinematic viscosity) encountered in any measurement was 5620 .

The mass ratio, $m^{*}$, is defined as the ratio of the cylinder mass to the displaced fluid mass, and the aspect ratio is equal to the axial length of the cylinder, $L$, divided by its diameter (i.e. its width in the transverse direction). The elliptical cylinders and the $m^{*}=0.98$ circular cylinder were manufactured using a 3D printer and were polished to reduce surface roughness. In each case the major axis was aligned perpendicular to the freestream velocity. The ratio of the major to minor axes is defined as the ellipticity ratio, $\epsilon$, where $\epsilon=1$ for a circle and $\epsilon \rightarrow \infty$ for a flat plate.

The velocity fields surrounding the cylinders were measured using ParticleImage Velocimetry. The plane in the centre span of the cylinder was illuminated using an Nd:Yag laser and image-pairs were acquired using a high speed CMOS camera with a resolution of $1024 \times 1280$ pixels. The flow was seeded using $10 \mu \mathrm{m}$ silver-coated hollow glass spheres. For each measurement at a given reduced velocity, 1000 image-pairs were acquired at $200 \mathrm{~Hz}$. The velocity fields were calculated using a three-pass cross-correlation scheme, with $50 \%$ window overlap, starting with an initial interrogation window size of $64 \times 64$ pixels and culminating with a final window size of $24 \times 24$ pixels. After each pass the median filter test (Westerweel and Scarano, 2005) 
was applied and vectors which failed the test were replaced with the local median. The percentage of vectors which failed the median test on the final pass was less than $1.5 \%$. The resolution of the PIV measurements varied between the 21.23 and 30.54 pixels $/ \mathrm{mm}$, depending on the experiment, as detailed in Table 2.

The $m^{*}=1.17$ circular cylinder was made of transparent acrylic, while all other cylinders were opaque (the triangles were made of black plastic while the 3D printed cylinders were made of nylon and spray-painted black). This meant that PIV could be performed accurately very close to the cylinder surface only in the $m^{*}=1.17$ case, while in other cases the reflections and light scatter at the cylinder surface reduced accuracy in this region.

Prior to each measurement, the flowrate was adjusted and the system was allowed at least ten minutes to settle. A traverse system was then used to raise the PIV system to acquire 50 velocity fields upstream of the cylinder, which were then averaged to measure the freestream velocity, before the system was lowered again to acquire measurements of the flow surrounding the cylinder. The cylinder displacement was measured directly from the PIV images using a template-matching cross-correlation scheme. The method is outlined in Cagney and Balabani (2013c) and and involves cropping a template image of the cylinder from a single PIV frame, cross-correlating the template with every image in a data set and using the peak in the crosscorrelation matrix to track changes in the cylinder position (similar to the approach used in PIV). Spline-fitting was applied to the region surrounding the peak of the cross-correlation matrix to improve the resolution. Tests in which the cylinder was physically displaced using gauges indicated that the method was accurate to within 0.4 pixels. The displacement signals were band-pass filtered with cut-off frequencies of $5 \mathrm{~Hz}$ and $40 \mathrm{~Hz}$, to reduce the influence of noise and low frequency fluctuations not associated with VIV. The vibration amplitude, A, was measured as the root-mean-square of the filtered signal.

The reference frame was defined with respect to the centre of the cylinder in the transverse direction $(y)$, and in the flow direction $(x)$ the origin was taken to be the widest point of the cylinder.

The vortex-shedding frequency was estimated from the temporal coefficient of the most energetic Proper-Orthogonal Decomposition (POD) mode (van Oudheusden et al., 2005), calculated over a subset of the PIV fields; $x / D=-1$ to 3 and $y / D=0$ to 1 . This method was found to be robust, regardless of the cylinder geometry or the wake mode occurring. This POD 
signal was also used to phase-averaged the set of instantaneous PIV measurements at each reduced velocity into 16 phases. The vorticity fields and streamlines were calculated from the phase-averaged fields using least-squares differentiation and the streamline function in Matlab, respectively.

\section{Results}

\subsection{Triangular Cylinders}

The variation in the amplitude response and the estimated vortex-shedding frequency are presented in Figure 3, for the $m^{*}=1.17$ circular cylinder and the two triangular cylinders. The circular cylinder exhibits the well-known two-branch response regime, with a reduction in amplitude at resonance $\left(U_{r} S t \approx 0.5\right)$, which has been described previously (Cagney and Balabani, 2013b,c; Okajima et al., 2004; Naudascher, 1987). The first branch occurs over $U_{r} S t \approx 0.25-0.5$, has an amplitude of $A / D \approx 0.065$ and is characterised by predominantly symmetric vortex-shedding that breaks down into an alternate arrangement downstream (Cagney and Balabani, 2013a; Konstantinidis et al., 2007). The symmetric shedding occurs at the cylinder oscillation frequency, and $f_{v s} / f_{n} \approx 1$ in this region.

The vibration amplitude is dramatically reduced at the centre of the response regime, before increasing again to form the second response branch, with a slightly lower peak amplitude of $A / D \approx 0.04$. This branch can occur in multiple forms (Cagney and Balabani, 2013b); in this case the SA mode is observed for $U_{r} S t=0.55$ (two vortices shed per wake cycle), and the A-IV mode is observed for $U_{r} S t=0.6-0.71$ (two vortex pairs shed per cycle). The doubling of the number of vortices shed per cycle leads to a doubling in the frequency of velocity fluctuations in the near wake (Figure 3(b)). The entire lock-in range, where the vortex-shedding occurs at either $\approx f_{n} / 2$ or $\approx f_{n}$ spans $U_{r} S t \approx 0.2-0.71$.

The upstream-pointing triangular cylinder exhibits a slight increase in vibration amplitude over the same reduced velocity range, $U_{r} S t \gtrsim 0.2$, but the amplitude is considerably lower, with $A / D \approx 0.02$. Figure 3(b) indicates that the vortex-shedding occurs at the Strouhal frequency and does not lockin to the structural vibration frequency, suggesting that the response is not associated with VIV. Likewise, the downstream-pointing cylinder does not exhibit lock-in, and the structural response is negligible.

The frequency response of the three cylinders is summarised in Figure 4, using stacks of the Fast Fourier Transform of the streamwise displacement 

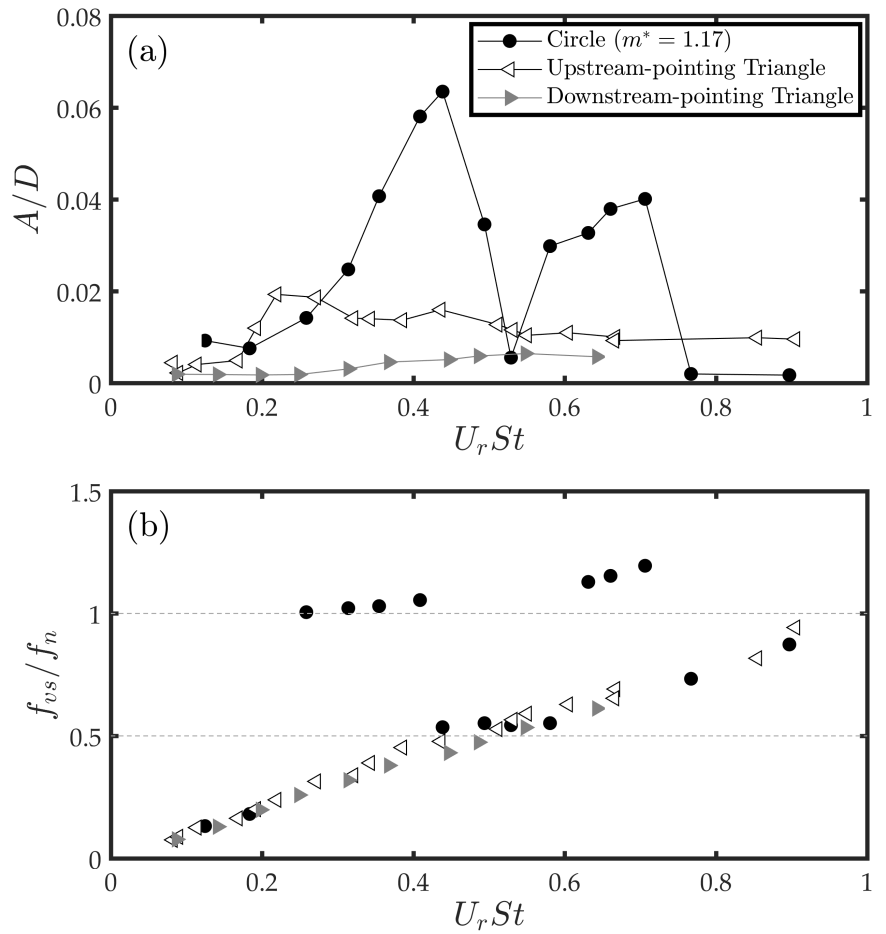

Figure 3: Variation in the amplitude response (a) and the vortex-shedding frequency (b) with reduced velocity, for a circular cylinder $\left(m^{*}=1.17\right)$ and two triangular cylinder $\left(m^{*}=1.4\right)$ pointing upstream/downstream. The shedding frequency is estimated from the transverse velocity signal measured at $x / D=1.5, y / D=0.5$. 

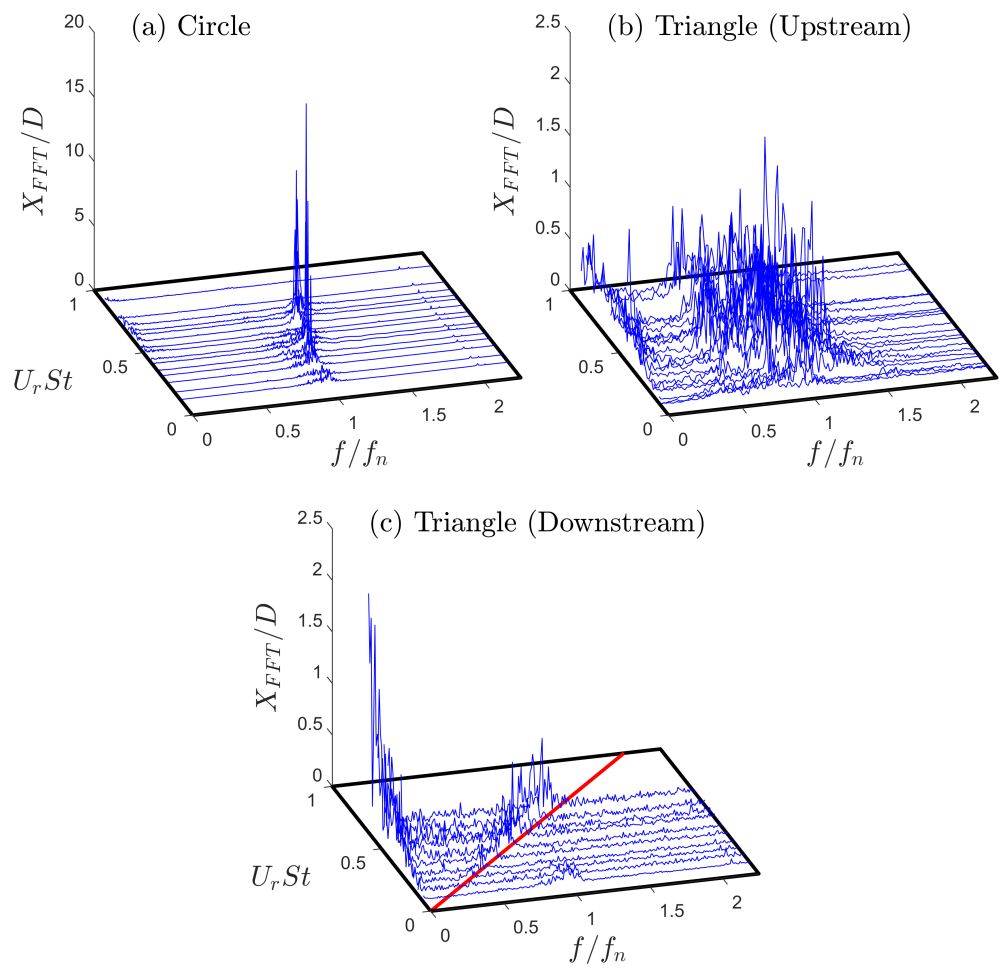

Figure 4: Stacks for Fast Fourier Transform spectra of the cylinder displacement signals for a range of reduced velocity for the circular cylinder $\left(m^{*}=1.17\right)$ (a), the upstream-pointing (b) and downstream-pointing triangular cylinders (c). The red line in (c) corresponds to the Strouhal frequency.

signal, $X_{F F T}$, calculated at each reduced velocity. The spectra for the circular cylinder tend to exhibit a single, narrow peak close to the natural frequency, and a minor harmonic at $2 f_{n}$. The peak response frequency increases slightly with reduced velocity due to added mass effects.

In contrast, the spectra for the upstream-pointing triangular cylinder are broadband (Figure 4(b)), with many peaks occurring in the range $f / f_{n} \approx$ $0-1.5$. There is no clear tendency for peaks to occur at either $f_{n}$ or the shedding frequency, which suggests that the cylinder vibrations are caused by turbulent buffeting, rather than a coherent, periodic process such as vortexshedding. 
Finally, the spectra for the downstream-pointing cylinder are shown in Figure 4(c). In this case, at high reduced velocity the spectra show peaks at low frequencies, and there does not appear to be any tendency for energy to occur at the natural frequency; instead, for $U_{r} S t \gtrsim 0.25$, the cylinder vibration frequency coincides with the Strouhal frequency (indicated by the red line).

The absence of vibration is a somewhat surprising result: although the upstream- and downstream-pointing cylinders both exhibit similar vibration amplitudes and vortex-shedding frequencies (Figure 3) and have apparent geometric similarities, their sets of spectra indicate that the nature of their dynamics are very different, with the former case being characterised by chaotic, broadband vibrations and the latter characterised by a tendency to lock-in to the shedding frequency. The shifting of the vibration frequency to match the fluid forcing frequency, rather than the shedding frequency locking in to $f_{x}$, is unusual for elastically mounted bodies (Sarpkaya, 2004; Williamson and Govardhan, 2004; Naudascher, 1987). It is also unusual that this cylinder does not exhibit significant vibrations, given that the vortexshedding (i.e. the excitation) and the structural response are synchronised.

This may be explained by considering the 'true' reduced velocity, $U_{r, t}$, which gives the predicted vortex-shedding frequency with respect to the actual vibration frequency and thus accounts for changes in $f_{x}$ due to added mass effects (Govardhan and Williamson, 2000):

$$
U_{r, t}=\frac{f_{S t}}{f_{x}}=U_{r} S t / f^{*}=0.5
$$

where $f^{*}=f_{x} / f_{n}$ is the frequency ratio (with $f_{x}$ found from the peaks in Figure 4(a)). This indicates that the shifting of the cylinder vibration frequency causes the cylinder to occupy the local minima in the streamwise response regime that occurs at $U_{r} S t \approx 0.5$ (see Figure 3(a) and Naudascher (1987)), and suggests that the physical mechanism responsible for the well known low amplitude region for the circular cylinder is also responsible for the absence of VIV of the downstream-pointing triangular cylinder.

Phase-averaged vorticity fields are shown in Figure 5 for the three cases, for reduced velocities corresponding to pre-lock-in and the first and second response branches. As has been shown in previous studies, the circular cylinder is associated with the von Kármán vortex street (A-II mode) pre-lock-in $\left(U_{r} S t \approx 0.1\right.$, Figure 5, left column), symmetric shedding that break down into an alternate pattern downstream for much of the first branch $\left(U_{r} S t \approx 0.35\right.$, 
$(\mathrm{a}, \mathrm{i})$ Circle $-U_{r} S t=0.124$

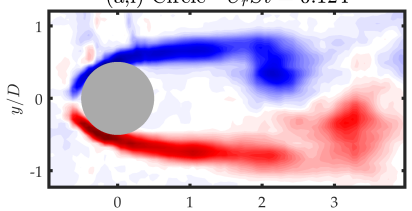

(b,i) Triangle - $U_{r} S t=0.0891$

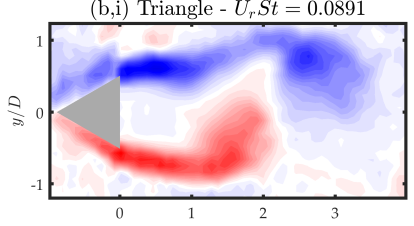

(c,i) Triangle $-U_{r} S t=0.087$

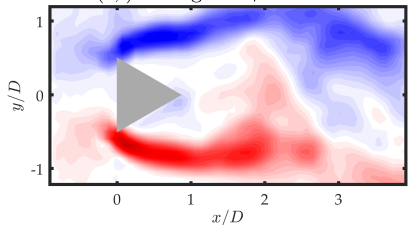

(a,ii) Circle $-U_{r} S t=0.355$

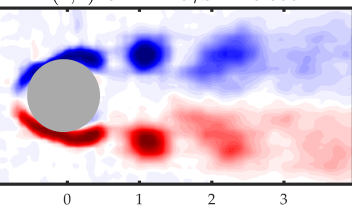

(b,ii) Triangle - $U_{r} S t=0.343$

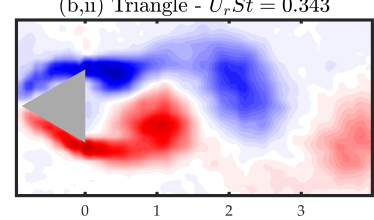

(c,ii) Triangle $-U_{r} S t=0.368$

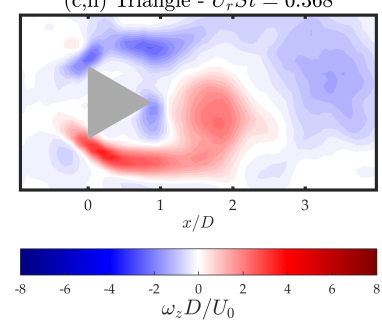

(a,iii) Circle $-U_{r} S t=0.66$

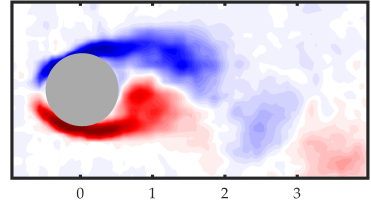

(b,iii) Triangle - $U_{r} S t=0.666$

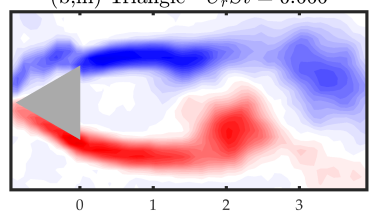

(c,iii) Triangle $-U_{r} S t=0.643$

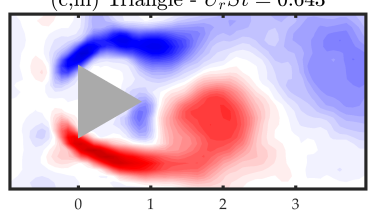

Figure 5: Phase-averaged vorticity fields for the circular cylinder $\left(m^{*}=1.17\right.$, top row $)$, the upstream-pointing (middle row) and the downstream-pointing triangular cylinders (bottom row). The left column corresponds to the pre-lock-in $\left(U_{r} S t \approx 0.1\right)$, the middle column corresponds to the region of the first response branch $\left(U_{r} S t \approx 0.35\right)$ and the right column corresponds to the second branch $\left(U_{r} S t \approx 0.6\right)$. The phases shown correspond to the point in the cycle at which the vorticity along the wake centre line reaches a maximum.

Figure 5, middle column), and alternately shed vortices (in this case the A-IV mode) in the second branch ( $U_{r} S t \approx 0.6$, Figure 5 , right column).

The vorticity fields for the triangular cylinders indicate that there is little noticeable change in the shedding process throughout this reduced velocity range. There is considerable vorticity present at the base of the downstreampointing cylinder, and it is therefore reasonable to expect that this point also coincides with significant pressure fluctuations. These enhanced pressure fluctuations on the cylinder surface are likely to increase the magnitude of the fluctuating drag force, and may explain why the fluctuating drag force is strong enough to shift the vibration frequency of the cylinder, even in the absence of significant VIV, as it is well known that the after body shape can have a significant effect on the fluid-structure interaction (Deniz and Staubli, 1997). 

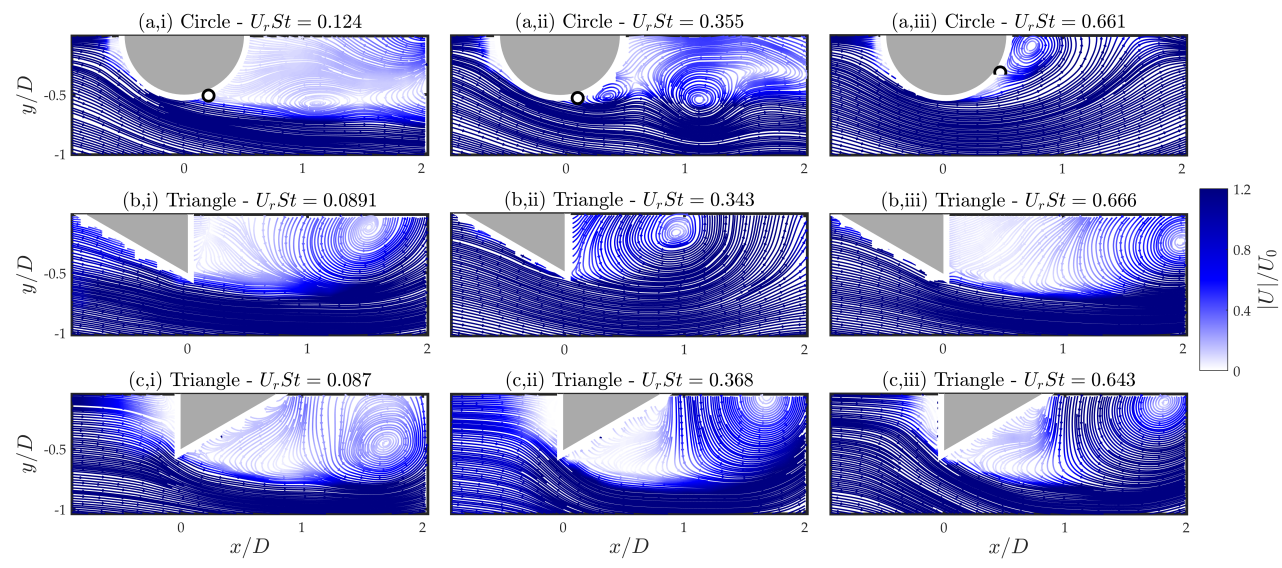

Figure 6: Streamlines calculated from the phase-averaged velocity fields for the circular cylinder $\left(m^{*}=1.17\right.$, top row), the upstream-pointing (middle row) and the downstreampointing triangular cylinders (bottom row). The left column corresponds to the pre-lock-in $\left(U_{r} S t \approx 0.1\right.$ ), the middle column corresponds to the region of the first response branch $\left(U_{r} S t \approx 0.35\right)$ and the right column corresponds to the second branch $\left(U_{r} S t \approx 0.6\right)$. The phases shown correspond to the point in the cycle at which the vorticity along the wake centreline reaches a maximum. Colours indicate the local velocity magnitude, $|U|$. Velocity data within $0.05 \mathrm{D}$ of the surface of the cylinder are neglected. 

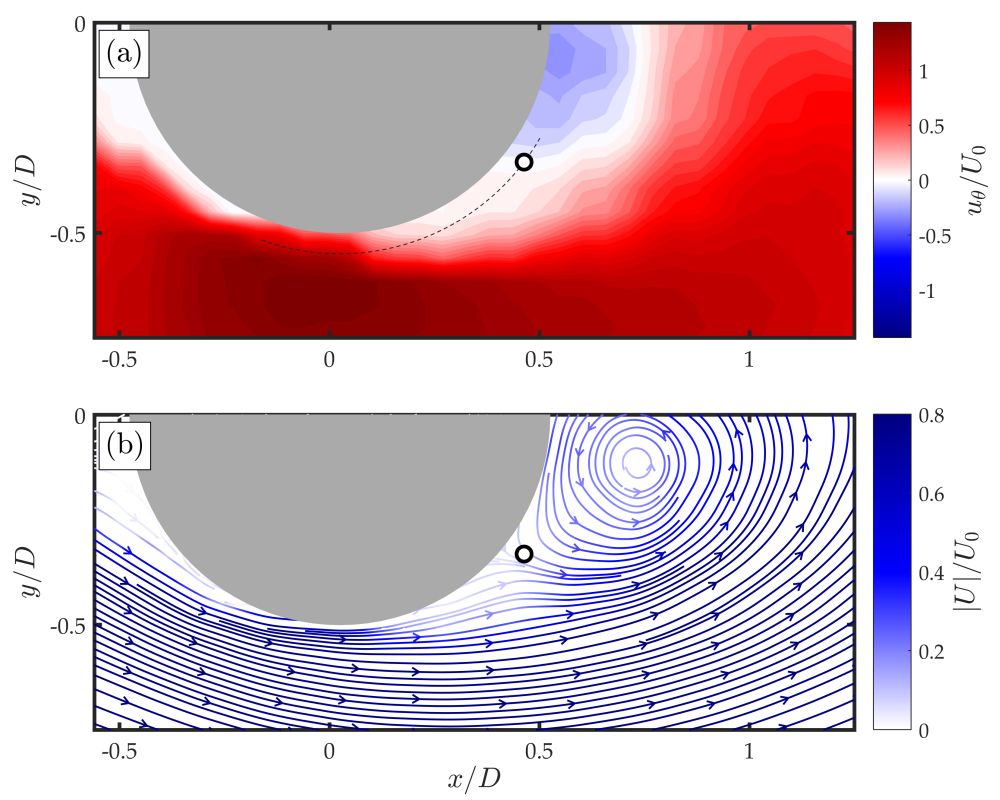

Figure 7: Demonstration of the method used to estimate the separation point surrounding the circular cylinder $\left(m^{*}=1.17\right)$ at $U_{r} S t=0.661$. The tangential velocity about the centre of the cylinder (a) and the streamlines at the same phase, with the colour indicating the local velocity magnitude (b). The dashed line in (a) at $r / D=0.55$ indicates where the tangential velocity was interpolated to find where $u_{\theta}=0$, which was taken as the separation point (indicated by the black and white circles).

The corresponding streamlines for each phase-averaged field are shown in Figure 6 . The colour of each streamline indicates the local velocity magnitude, $|U|=\sqrt{u^{2}+v^{2}}$. Due to the difficulty in resolving the flow field close to opaque boundaries using PIV (Raffel et al., 2007), vectors within $0.05 D$ of the cylinder surface are neglected. However, the transparency of the acrylic circular cylinder $\left(m^{*}=1.17\right)$ allows the PIV measurements to resolve the flow close to the cylinder, as can be seen in Figure 6(a).

This can be used to estimate the separation point at a given phase, by taking advantage of the fact that at the separation point the tangential velocity (parallel to the surface) goes to zero. The technique is demonstrated in Figure 7 for a single reduced velocity and phase. The Cartesian velocity vectors are converted into the tangential velocity about the centre of the cylinder, $u_{\theta}$ (Figure $7(\mathrm{a})$ ), which is then interpolated along the arc at a dis- 
tance $r / D=0.55$ from the cylinder centre (dashed grey line). The point where the interpolated tangential velocity is equal to zero is identified as the separation point (indicated by the black and white marker). This approach was found to be robust and was consistent with the streamlines found for the same phase, as can be seen in Figure 7(b).

The three sets of streamlines in Figure 6 indicate that location of the separation point varies with reduced velocity and the dominant wake mode. In contrast, even allowing for the uncertainty near solid boundaries, the streamlines for the triangular cylinder measurements indicate that separation always occurs at the sharp corners. This was observed for all reduced velocities and phases examined.

This suggests that the ability of the separation point to shift may play an important role in the fluid-structure interaction. To further investigate this, the separation point was identified from the phase-averaged velocity fields of the circular cylinder wake for each phase and for each reduced velocity. The resulting separation angles, $\theta_{s p}$, measured from the upstream stagnation point of the cylinder, are presented in Figure 8(a), along with the average angle found at each reduced velocity.

The presence of significant amplitude vibrations coincides with an increase in the mean separation angle (i.e. the separation point moves further downstream), which is particularly evident at the peaks in the vibration amplitude in the first and second response branches, at $U_{r} S t \approx 0.3-0.45$ and $0.55-0.71$, respectively. There is also a decrease in $\theta_{s}$ at the low amplitude region at $U_{r} S t \approx 0.5$. The mean value of $\theta_{s p}$ when the cylinder is not undergoing VIV appears to decrease slightly with reduced velocity, as can be seen by comparing the data points pre- and post-lock-in, which may be the result of Reynolds number effects or changes in the cylinder response characteristics.

It is also notable that VIV is associated with an increase in the variation in the separation angle throughout the shedding cycle. When the cylinder vibration amplitude is low pre-lock-in $\left(U_{r} S t \leq 0.25\right)$, at the centre of the response regime between the two branches $\left(U_{r} S t \approx 0.5\right)$ and post-lock-in $\left(U_{r} S t \geq 0.72\right)$, the separation angles show very little variation.

This intra-cycle variation of $\theta_{s p}$ is demonstrated in Figure 9, which shows how the separation angle varies throughout the shedding cycle at the peak of the first branch, in the low amplitude region between the two branches, in the second response branch and post-lock-in. It is clear that when the cylinder vibration amplitude is low, the separation angle remains relatively constant, 

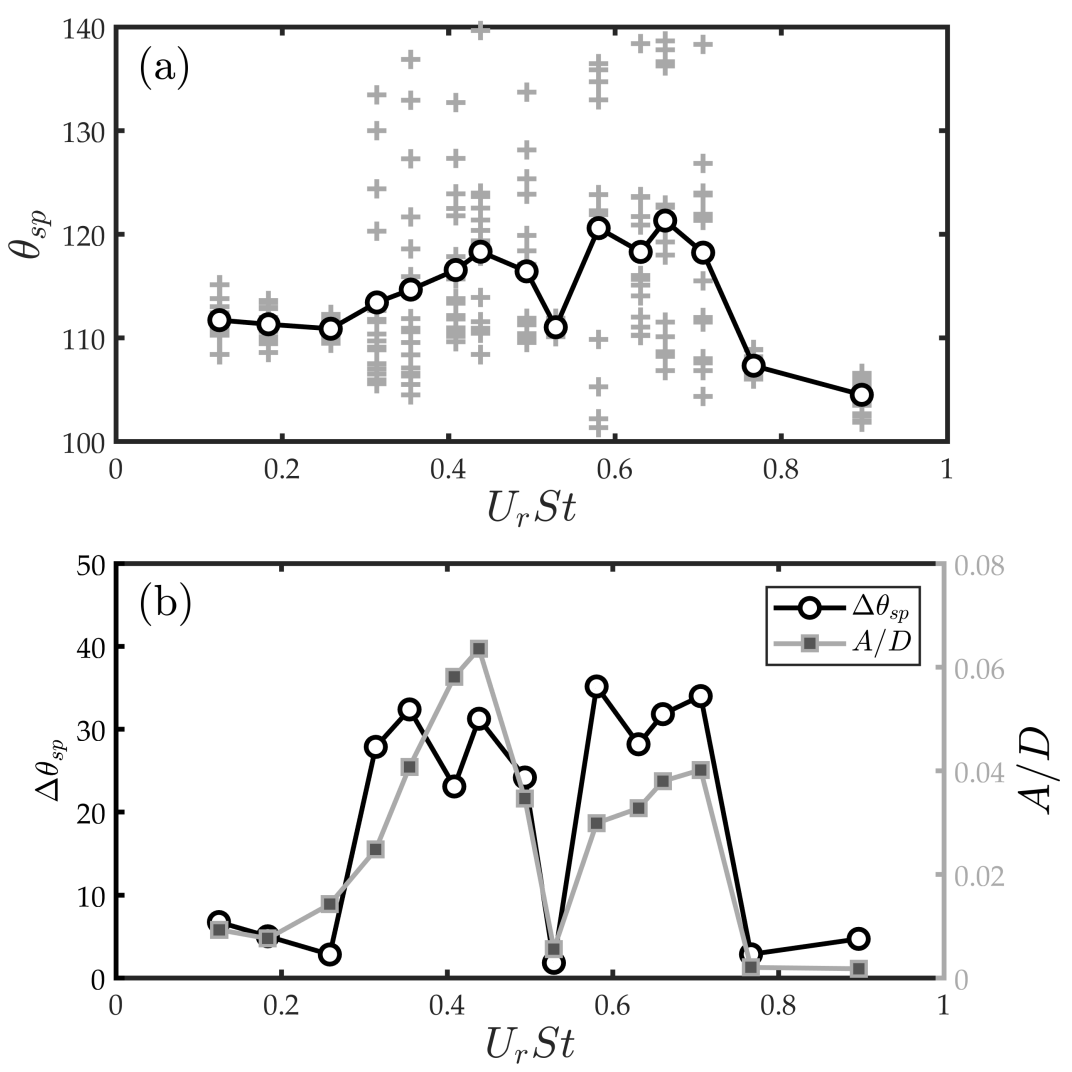

Figure 8: Variation in the separation angle (grey crosses) with reduced velocity for the circular cylinder (a). The grey crosses represent the values of $\theta_{s p}$ found for each of the 16 phase-averaged fields, and the black and white circles denote the average angle found at each reduced velocity. The range of angles (i.e. the different between the maximum and minimum found at a given reduced velocity measurement) and the cylinder amplitude response are shown in (b). 


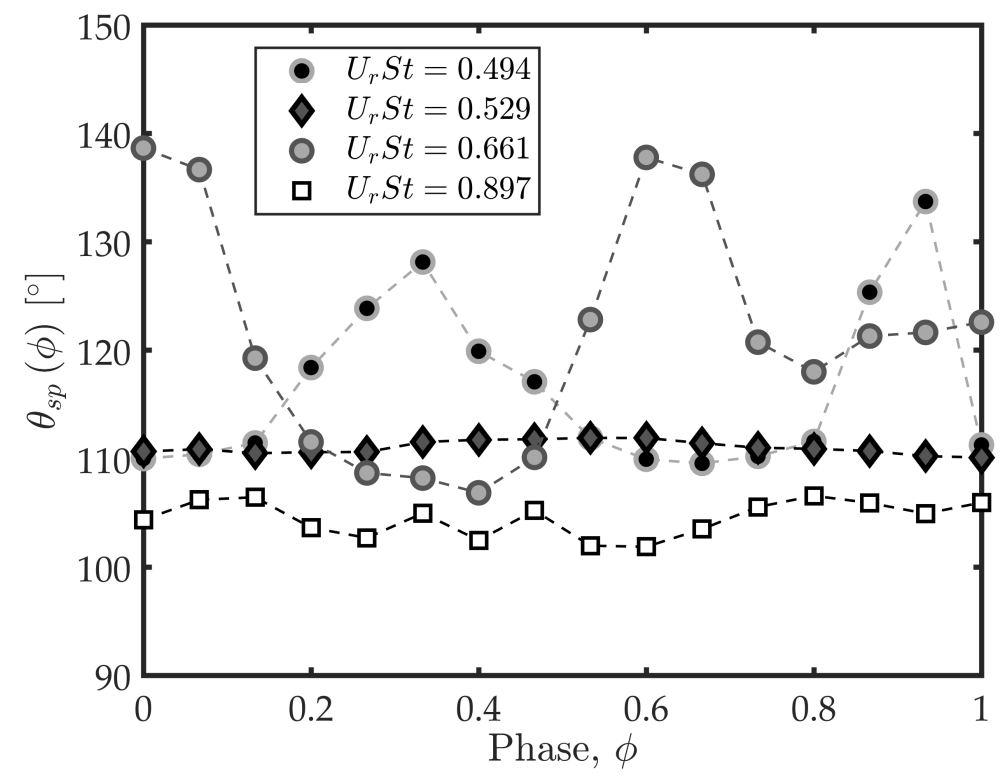

Figure 9: Variation in the separation angle throughout the shedding cycle for four values of reduced velocity, corresponding to a point in the first response branch $\left(U_{r} S t=0.494\right.$, grey and black circles), the low amplitude region between branches $\left(U_{r} S t=0.529\right.$, diamonds), the second response branch $\left(U_{r} S t=0.661\right.$, grey circles $)$, and post-lock-in $\left(U_{r} S t=0.897\right)$, open squares. 
while when VIV is occurring it varies throughout the shedding cycle. This also indicates that the scatter in the data plotted in Figure 8 is physical and represents the intra-cycle variation in the separation angle, rather than merely being caused by uncertainties in the method of estimating $\theta_{s p}$.

The magnitude of the intra-cycle variation in the separation angle, $\Delta \theta_{s p}$, is summarised in Figure 8(b), where $\Delta \theta_{s p}$ is the difference between the max-

imum and the minimum values of $\theta_{s p}$ encountered throughout a shedding cycle. There is a very clear correlation between $\Delta \theta_{s p}$ and the vibration amplitude. It is known from previous studies (Naudascher, 1987; Okajima et al., 2004; Cagney and Balabani, 2013c) and is evident in the present data that the von Kármán vortex street, in which the vortices form a wake pattern very similar to that seen behind stationary cylinders, is not capable of driving self-sustaining vibrations. For streamwise VIV, a modified shedding process is required, involving either a different wake pattern (e.g. the S-I or A-IV modes), or a significant reduction in the vortex-formation length and vortex trajectories and an increase in the strength of the shed vortices, as occurs for the SA mode or at the peak of the first branch (Cagney and Balabani, 2013c). Figure 8 indicates that the near wake dynamics associated with the various wake modes that are capable of exciting stable VIV require a variation in the separation point, at least to initiate vibration. This may explain the absence of VIV for the triangular cylinder cases, even during lock-in; the sharp corners of the triangular cylinders act as fixed separation points and do not allow $\theta_{s p}$ to vary, preventing the development of wake modes that are capable of inducing streamwise VIV.

\subsection{Elliptical Cylinders}

We have observed that circular cylinders with no fixed separation points exhibit VIV, while triangular cylinders with sharp corners that act as fixed separation points, do not, despite having similar structural properties. It is therefore interesting to examine an intermediate case; elliptical cylinder, which maintain the transverse symmetry of the circular and triangular cylinders, but whose separation point can be gradually varied from fixed (large ellipticity ratio, $\epsilon \gg 1)$ to not fixed $(\epsilon=1)$.

The elliptical cylinder examined had a lower mass ratio than the cylinder discussed in the previous section, and therefore it was decided to perform measurements with a reference case of a circular cylinder $(\epsilon=1)$ with the same mass ratio. The amplitude response and the vortex-shedding frequency 

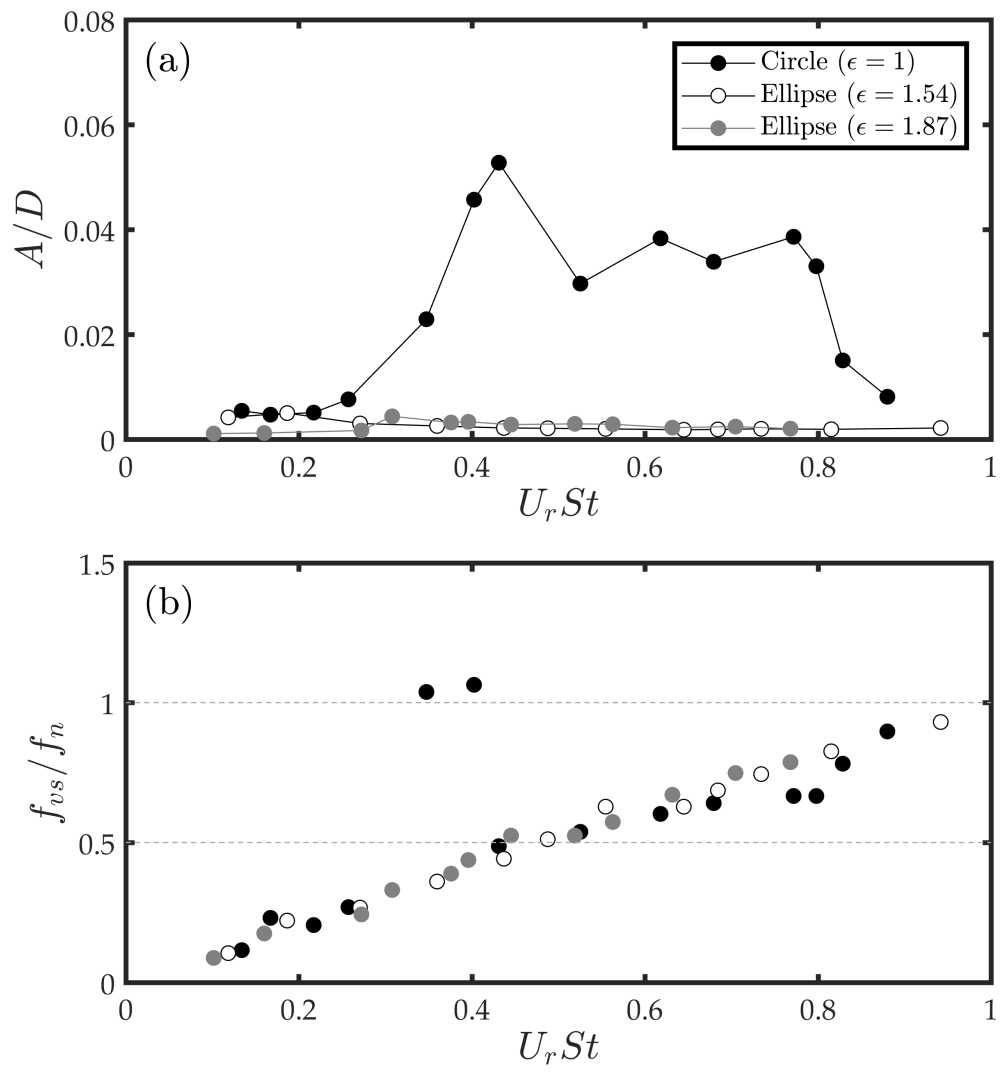

Figure 10: Variation in the amplitude response (a) and the vortex-shedding frequency (b) with reduced velocity, for a circular cylinder and two elliptical cylinder $(\epsilon=1.5$ and 1.9). The shedding frequency is estimated from the transverse velocity signal measured at $x / D=1.5, y / D=0.5$. The mass ratio of each cylinder is $m^{*}=0.98$. 

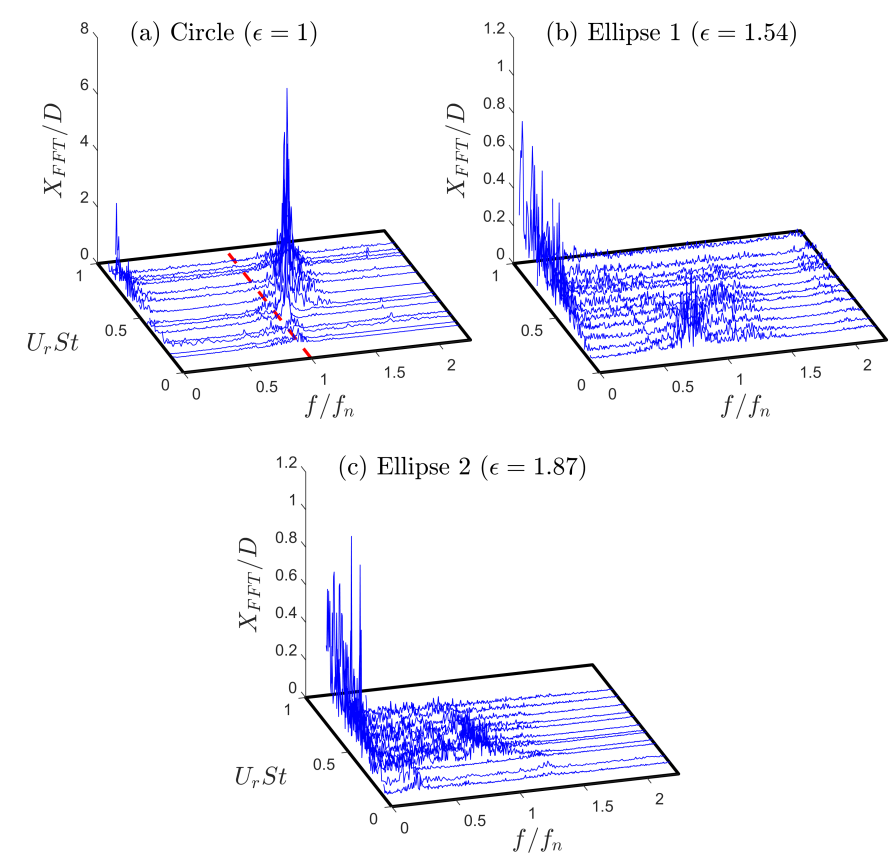

Figure 11: Stacks of Fast Fourier Transform spectra of the cylinder displacement signals for a range of reduced velocity for a circular and elliptical cylinders: (a), $\epsilon=1 ;$ (b), $\epsilon=1.54 ;$ and $\epsilon=1.87$. The dashed red line in (a) denotes the natural frequency.

are shown in Figure 10(a) and 10(b), respectively, and stacks of the spectra of the cylinder displacement signals are presented in Figure 11.

Despite the relatively small difference in the mass ratio compared to the acrylic cylinder discussed in the previous section (less than 21\%), the response regime of the low mass ratio circular cylinder presented in Figure 10(a) shows some significant differences from the results presented in Figure 3(a). The first response branch is relatively unchanged, but the low amplitude region at $U_{r} S t \approx 0.5$ is no longer apparent and the second response branch is less clearly defined, spanning a greater reduced velocity range (up to $U_{r} S t \approx$ 0.8). Examination of the spectra of the displacement signals in Figure 11(a) indicate that the latter feature is due to an increase in the vibration frequency due to added mass effects, which have an enhanced effect on the vibration frequency of low mass ratio structures (Govardhan and Williamson, 2000). This leads to an increased vibration frequency of approximately $40 \%$ at the 
highest reduced velocity relative to the natural frequency (red line in Figure 11(a)). As a result, even though Figure 10(b)) shows the velocity fluctuations in the near wake occurring at $f_{v s} / f_{n} \approx 0.7$, in the region of the second branch $\left(U_{r} S t \approx 0.6-0.8\right)$, these still approximately coincide with half the cylinder vibration frequency, indicating that lock-in is still occurring (i.e. $\left.f_{v s} / f_{x}=0.5\right)$.

The spectra peaks are broader than those observed for the higher mass ratio case (Figure 4(a)). This is a result of the greater complexity of the coupling between the structural dynamics and the wake, as the low $m^{*}$ cylinder is more susceptible to unsteady added mass effects and the resulting fluctuations in the cylinder response frequency. This in turn leads to enhanced feedback between the flow and the structural motion relative to higher $m^{*}$ cases and causes the displacement signals to become more variable and less periodic.

In spite of these variations and added mass effects, the salient features of the streamwise VIV response regime can still be observed: two response branches can be identified; the cylinder vibration amplitude is of the order $A / D \sim 0.1$; the vortex-shedding becomes locked in to the cylinder vibration frequency; and the spectra of the cylinder displacement signals tend to have a single peak close to or above $f_{n}$.

None of these features are observed for the elliptical cylinder cases $(\epsilon=$ 1.54 and 1.87): the amplitude response is negligible $(A / D<0.01)$ and the vortex-shedding occurs at the Strouhal frequency for the entire reduced velocity range examined. The spectra of the displacement signals (Figures 11(b) and $11(\mathrm{c}))$ are very broad, containing several small peaks for $f / f_{n} \lesssim 1.5$, with most energy occurring at very low frequencies. This is similar to that observed for the upstream-pointing triangular cylinder (Figure 4), and suggests that the low amplitude vibrations that the elliptical cylinders experience are associated with turbulent buffeting rather than VIV.

The absence of VIV for the elliptical cylinders and their broadband spectra cannot be attributed to their structural properties, as the circular cylinder with the same mass ratio and similar structural damping clearly exhibited a VIV response. These results indicate that even a cylinder with a moderate ellipticity of $\epsilon=1.54$ is sufficiently oblate to inhibit the movement of the separation point and suppress VIV.

This is in contrast to the findings of Navrose et al. (2014) and Hasheminejad and Jarrahi (2015), who observed that at low Reynolds numbers $(R e<400)$ that in the transverse response regime $\left(U_{r} S t>0.7\right)$ the struc- 
tural vibrations in both the streamwise and transverse directions were increased for $\epsilon>1$. This may be a consequence of the difference in Reynolds number, as some wake modes are known to only occur at high Re (Leontini and Thompson, 2013). Alternatively, the fact that the ellipticity ratio reduces streamwise VIV but promotes VIV occurring in primarily the transverse direction may reflect fundamental differences in the vortex-shedding and fluid-structure interaction for streamwise and transverse VIV.

The data presented here demonstrate the very high sensitivity of streamwise VIV response of cylinders to the ability of the separation points to move. This is consistent with previous studies examining the streamwise response of flat plates aligned almost perpendicular to the flow and diamond-shaped cylinders (Naudascher, 1987), and indicates that the location and movement of the separation point plays a greater role in the complex fluid-structure interaction governing streamwise VIV than has previously been acknowledged.

\section{Conclusions}

Particle-Image Velocimetry measurements were acquired of the flow field surrounding cylinders of various cross-sectional shapes that were free to move only in the streamwise direction. It was found that two circular cylinders with slightly different mass ratios exhibited VIV, with a response regime dominated by two branches, as has been found in several previous studies in the literature. However, none of the non-circular cylinders underwent any significant VIV, even when the vortex-shedding frequency coincided with the natural frequency of the structure. The absence of VIV could not be attributed to differences in the structural properties such as damping, mass ratio, aspect ratio etc. or flow conditions such as Reynolds number or blockage ratio. Instead, the appearance or absence of fluid excitation appeared to arise as a results of the fixed nature of the separation point for triangular and elliptical cylinders.

Examination of the near wake of the circular cylinder $\left(m^{*}=1.17\right)$ indicated that when the cylinder exhibited VIV, the separation point varied by approximately $30^{\circ}$ over the course of a single shedding cycle, while the separation angle was approximately constant for cases when the vibration angle was low. It was argued that the ability of the separation point to move was a requirement for the various wake modes that are capable of exciting streamwise VIV, and that the absence of VIV for the triangular cylinder was a result of the sharp corners and the fixed nature of the separation point. 
In order to examine intermediate cases where the separation point was more restricted than in the circular cylinder case, but less restricted than in the triangular cylinder case, the response regimes of elliptical cylinders aligned with the major axis perpendicular to the flow were studied. These cylinders had a low mass ratio and were thus highly susceptible to added mass effects and temporal variations in response characteristics. Significant VIV was again observed for the circular case $(\epsilon=1)$, but not for the elliptical cylinders, even when the degree of ellipticity was moderate $(\epsilon=1.54)$.

The data presented in this study indicate that streamwise VIV is highly sensitive to the location of the separation point and its ability to move, which suggests that fixing the separation point may be an effective strategy to suppress VIV in this direction and thus raise the critical reduced velocity for the onset of VIV in multi-degree of freedom structures.

\section{References}

Aguirre, J.E., 1977. Flow induced, in-line vibrations of a circular cylinder. Ph.D. thesis. Imperial College of Science and Technology.

Ajith Kumar, R., H., S.C., Lakshmana Gowda, B.H., 2009. Influence of corner radius on the near wake structure of a transversely oscillating square cylinder. Journal of Mechanical Science and Technology 23, 2390 - 2416.

Bearman, P., Brankovicć, M., 2004. Experimental studies of passive control of vortex-induced vibration. European Journal of Mechanics - B/Fluids $23,9-15$.

Bernitsas, M.M., Raghavan, K., Ben-Simon, Y., Garcia, E.M.H., 2008. VIVACE (Vortex Induced Vibration Aquatic Clean Energy): A new concept in generation of clean and renewable energy from fluid flow. Journal of Offshore Mechanics and Arctic Engineering 130, 1-15.

Blevins, R.D., 1977. Flow-Induced Vibrations. Van Nostrad Reinhold.

Bokaian, A.R., Geoola, F., 1984. Hydroelastic instabilities of square cylinders. Journal of Sound and Vibration 92, 117-141.

Cagney, N., Balabani, S., 2013a. Mode competition in streamwise-only vortex induced vibrations. Journal of Fluids and Structures 41, 156-165. 
Cagney, N., Balabani, S., 2013b. On multiple manifestations of the second response branch in streamwise vortex- induced vibrations. Physics of Fluids $25,1-17$.

Cagney, N., Balabani, S., 2013c. Wake modes of a cylinder undergoing free streamwise vortex-induced vibrations. Journal of Fluids and Structures $38,127-145$.

Cagney, N., Balabani, S., 2014. Streamwise vortex-induced vibrations of cylinders with one and two degrees of freedom. Journal of Fluid Mechanics $758,702-727$.

Cagney, N., Balabani, S., 2016. Fluid forces acting on a cylinder undergoing streamwise vortex-induced vibrations. Journal of Fluids and Structures $62,147-155$.

Chen, J.M., Liu, C.H., 1999. Vortex shedding and surface pressures on a square cylinder at incidence to a uniform air stream. International Journal of Heat and Fluid Flow 20, 592-597.

Deniz, S., Staubli, T., 1997. Oscillating rectangular and octogonal profiles: interaction of leading- and trailing-edge vortex formation. Journal of Fluids and Structures 11, 3-31.

Govardhan, R., Williamson, C.H.K., 2000. Modes of vortex formation and frequency response of a freely vibrating cylinder. Journal of Fluid Mechanics $420,85-130$.

Griffin, O.M., Ramberg, S.E., 1976. Vortex shedding from a cylinder vibrating in line with an incident uniform flow. Journal of Fluid Mechanics 75, $257-271$.

Hasheminejad, S.M., Jarrahi, M., 2015. Numerical simulation of two dimensional vortex-induced vibrations of an elliptic cylinder at low Reynolds numbers. Computers \& Fluids 107, 25-42.

Jauvtis, N., Williamson, C.H.K., 2003. Vortex-induced vibration of a cylinder with two degrees of freedom. Journal of Fluids and Structures 17, 10351042. 
Jauvtis, N., Williamson, C.H.K., 2004. The effect of two degrees of freedomon vortex-induced vibration at low mass and damping. Journal of Fluid Mechanics 509, 22-63.

Konstantinidis, E., Balabani, S., 2007. Symmetric vortex shedding in a near wake of a circular cylinder due to streamwise perturbations. Journal of Fluids and Structures 23, 1047-1063.

Konstantinidis, E., Balabani, S., Yianneskis, M., 2005. The timing of vortex shedding in a cylinder wake imposed by periodic inflow perturbations. Journal of Fluid Mechanics 543, 45-55.

Konstantinidis, E., Balabani, S., Yianneskis, M., 2007. Bimodal vortex shedding in a perturbed cylinder wake. Physics of Fluids 19, 011701 1-4.

Leontini, J.S., Griffith, M.D., Lo Jacono, D., Sheridan, J., 2018. The flowinduced vibration of an elliptical cross-section at varying angles of attack. Journal of Fluids and Structures 78, 356-373.

Leontini, J.S., Thompson, M.C., 2013. Vortex-induced vibration of a diamond cross-section: Sensitivity to corner sharpness. Journal of Fluids and Structures 39, 371-390.

Levy, B., Liu, Y., 2013. The effects of cactus inspired spines on the aerodynamics of a cylinder. Journal of Fluids and Structures 39, 335-346.

Nakamura, A., Okajima, A., Kosugi, T., 2001. Experiments on flow-induced in-line oscillation of a circular cylinder in a water tunnel (2nd report, influence of aspect ratio of a cantilevered circular cylinder). JSME International Journal 44 (4), 705-711.

Naudascher, E., 1987. Flow-induced streamwise vibrations of structures. Journal of Fluids and Structures 1, 265-298.

Navrose, Vogeswaran, V., Sen, S., Mittal, S., 2014. Free vibrations of an elliptic cylinder at low Reynolds numbers. Journal of Fluids and Structures $51,55-67$.

Nemes, A., Zhao, J., Lo Jacono, D., Sheridan, J., 2012. The interaction between flow-induced vibration mechanisms of a square cylinder with varying angles of attack. Journal of Fluid Mechanics 710, 102-130. 
Nishihara, T., Kaneko, S., Watanabe, T., 2005. Characteristics of fluid dynamic forces acting on a circular cylinder oscillating in a streamwise direction and its wake patterns. Journal of Fluids and Structures 20, 505-518.

Odahara, S., Murakami, Y., Unoue, M., Sueoka, A., 2005. Fatigue failure by in-line flow-induced vibration and fatigue life evaluation. JSME International Journal Series A 48, 109-117.

Okajima, A., Nakamura, A., Kosugi, T., Uchida, H., Tamaki, R., 2004. Flowinduced in-line oscillation of a circular cylinder. European Journal of Mechanics B/Fluids 23, 115-125.

Ongoren, A., Rockwell, D., 1988. Flow structure from an oscillating cylinder Part 2. Mode competition in the near wake. Journal of Fluid Mechanics $191,225-245$.

van Oudheusden, B.W., Scarano, F., Hinsberg, N.P., Watt, D.W., 2005. Phase-resolved characterization of vortex shedding in the near wake of a square-section cylinder at incidence. Experiments in Fluids 39, 86-98.

Raffel, M., Willert, C., Wereley, S., Kompenhans, 2007. Particle Image Velocimetry. 2 ed., Springer.

Sarpkaya, T., 2004. A critical review of the intrinisic nature of vortex-induced vibrations. Journal of Fluids and Structures 19, 389-447.

Sen, S., Mittal, S., 2011. Free vibration of a square cylinder at low Reynolds numbers. Journal of Fluids and Structures 27, 875-884.

Sui, J., Wang, J., Liang, S., Tian, Q., 2016. VIV suppression for a large mass-damping cylinder attached with helical strakes. Journal of Fluids and Structures 62, 125-146.

Westerweel, J., Scarano, F., 2005. Universal outlier detection for PIV data. Experiments in Fluids 39, 1096-1100.

Williamson, C.H.K., Govardhan, R., 2004. Vortex-induced vibrations. Annual Review of Fluid Mechanics 36, 413-455.

Zdravkovich, M.M., 1981. Review and classification of various aerodynamic and hydrodynamic means for suppressing vortex shedding. Journal of Wind Engineering and Industrial Aerodynamics 7, 145-189. 